\title{
PLC Based Overcurrent Protection of Three-phase Transmission Line
}

\author{
Ahmed M. T. Ibraheem ${ }^{1}, \quad$ Mohammed A. Ibrahim ${ }^{2}, \quad$ Abdullah K. Shanshal ${ }^{3}$ \\ Ahmed_alnaib85@yahoo.com, mohammed.a.ibrahim1981@gmail.com, abd_eng83@yahoo.com \\ Dep. of Electrical Power Technologies Eng., Eng. Technical College/ Mosul, Northern Technical \\ University, Iraq 1,2 , and 3
}

\begin{abstract}
As the Transmission Line (TL) is an important part in the power system; this paper presents the design and practical implementation of overcurrent protection for threephase TL system using Programmable Logic Control (PLC). The PLC then on-line monitring the vlaue of the load current in each phase and detect the overcurrent and simaltinouslly isolate the fault by sending an output signal for tripping the coil of the Circuit Breaker (CB). Display unit of the PLC is used for displaying the load current, in addition to alert maessage with the type of the occoured fault. The proposed contrroller program also cancel of the trip signal of the CB during a definite time of the inrush and stating load current. In addition, an automatic reclosing system for return the CB to the work when the overcurrent is released. PLC-based protection methods cost less, provides greater precision with a safe way of operation when compared with the other protection controllers system. Function Block Diagram (FBD) language is employed for implementing the proposed software controller. Simulation is made for the proposed controller via LOGO! Soft Comfort V7.0 software programs for virtual implantation of the program before downloading to the PLC.
\end{abstract}

Keywords: Programmable Logic Control, Current Transformer, Precision rectifier, Protection System, Transmission Line, Circuit Breaker, and Function Block Diagram.

\section{Introduction}

Transmission and distribution lines are an important part of the power system as it has a good contribution in the generating unit and consumers to attain the continuity of electrical supply. Due to natural reasons as wind, rain, light storm the faults can happen in TLs. In general, faults can be categorized as shunt faults and series faults. Shunt faults are of two types; symmetrical and unsymmetrical faults. There are many possible shunt faults (L-G, LL-G, LLLG, LL, and LLL) that can occur in a three-phase TL. Among these shunt faults, three-phase(s) to phase (LLL) and three-phase(s) to ground faults (LLL-G) faults are considered as symmetrical faults whereas other shunt faults are counted as unsymmetrical faults [1]. Components of an electrical power system as TL can be protected using some elements, such as timers, contractors, voltage, and current relays. This mode is known as the "Classical Mode" which is very basic and contains mechanical dynamic parts [2]. Protection system based on PIC16F877A [3, 4], Arduino Microcontroller [5], FPGA [6] have eliminated many mechanical 
components. But this type of protection method requires an Analog to Digital Conversion (ADC) module, in addition to the PIC-based protection method does not visualize the electrical parameters measured. PLCs are the most important components embedded in industrial control systems, as they have less complexity, cost-efficient, simplicity and modularity, robustness and highly durable, and they require low maintenance [7].

In this study, the protection method For TLs based on a PLC is introduced. Moreover, the values of load currents for the three phases, and the faults that occurred in the system are on line monitored and alert messages are displayed on the PC desktop, as well as the PLC built-in display unit.

\section{Proposed Protection System Based on PLC}

The block diagram of the proposed system is shown in figure 1. It consists four major parts: the first part presented by three CTs, the second is three of Signal Conditioning Circuits (SCCs) for calibration the current signal of the CT to a suitable voltage signal to be injected to PLC unit, the third part is the PLC, and the final part is the CB; Three-phase conductor is used as a CB in this experimental work.

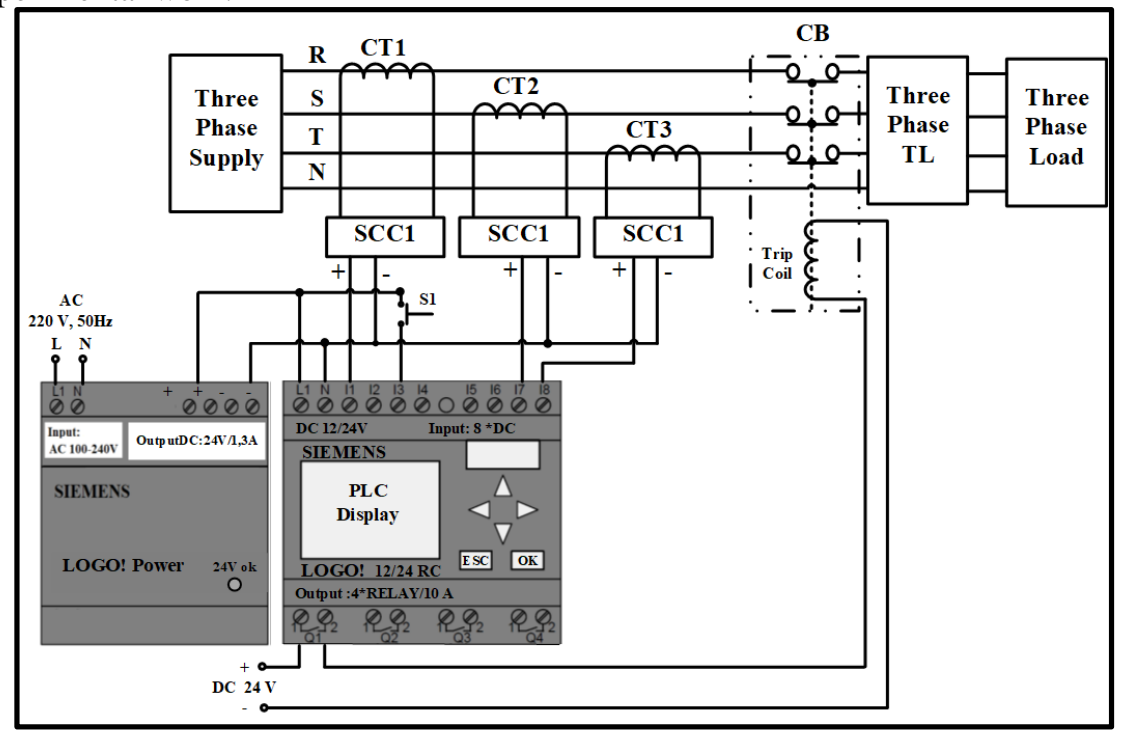

Fig. 1: Block diagram of the proposed protection system based on PLC

A CT is used for the measurement of electric currents. When the current in the circuit is too high to directly apply to the measurement instruments, therefore, it reduced. CT produces a reduced current accurately proportional to the current in the circuit. The CT with turn's ratio equal to (60:5) is used for each one of the three phase's power supplied to the TL.

Actually, the PLC can't even sense the current signal. So; a shunt resistor (Rsh) (also called as burden resistor) is fixed across the secondary windings (S1, and S2) of the CT as shown in figure 2. This resistor will not only make up the closed-loop but also converts the current signal of the CT to a voltage signal according Ohm's Law. Thus the produced voltage signal equivalent to the primary load current divided by the turn's ratio and multiplied by the value of R1. 
Three of SCCs are used for normalizing the converter voltage signal of the CT to voltage to the PLC analog voltage range to be measured. Each of SCC consists of a precision rectifier circuit based on operational amplifiers as shown in figure 2. This AC voltage is rectified by the first operational amplifier and then amplified by the second operational amplifier.

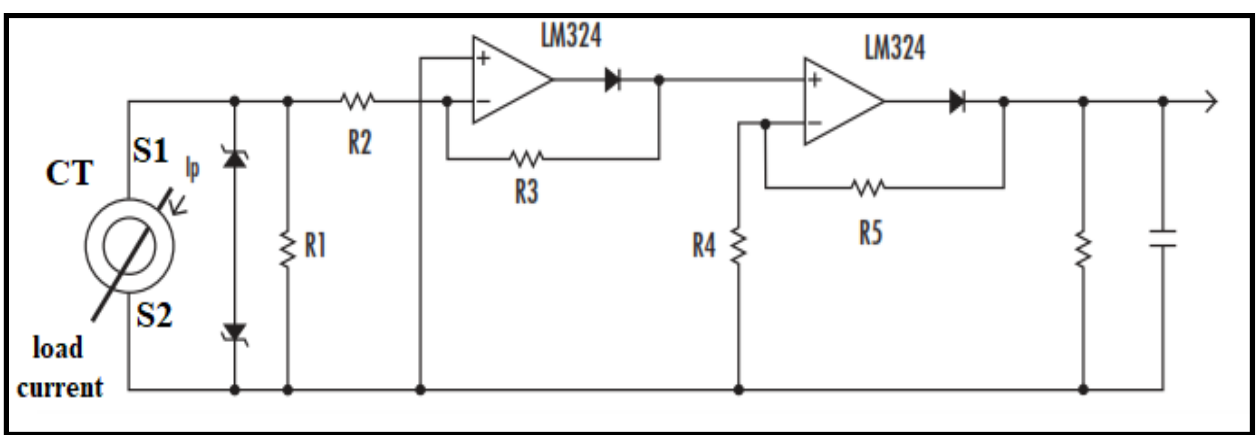

Fig. 2: Signal conditioning circuit for CT

Creating DC signal from an AC source will be associated with many problems such as diode voltage drops and the changes vs temperature and current. Therefore, this circuit offers a precise way of creating this DC signal.

The PLC that is used in the work is "LOGO! 12/24RC" (Version: 0BA6 [8]) and known as "LOGO! Basic"; which has 12/24 V DC input voltage, has eight digital inputs and four relays (10A) as digital outputs, LOGO! Power module (24 VDC, 1.3A) used as power supply. There is a special cable known as "LOGO!-PC cable" connected between the PLC unit and computer for downloading the control program.

\section{PLC Proposed Control Program for Protection System}

For PLC software, FBD method is used to perform the proposed control program using LOGO! Soft Comfort V7.0 as shown in figure 3. 


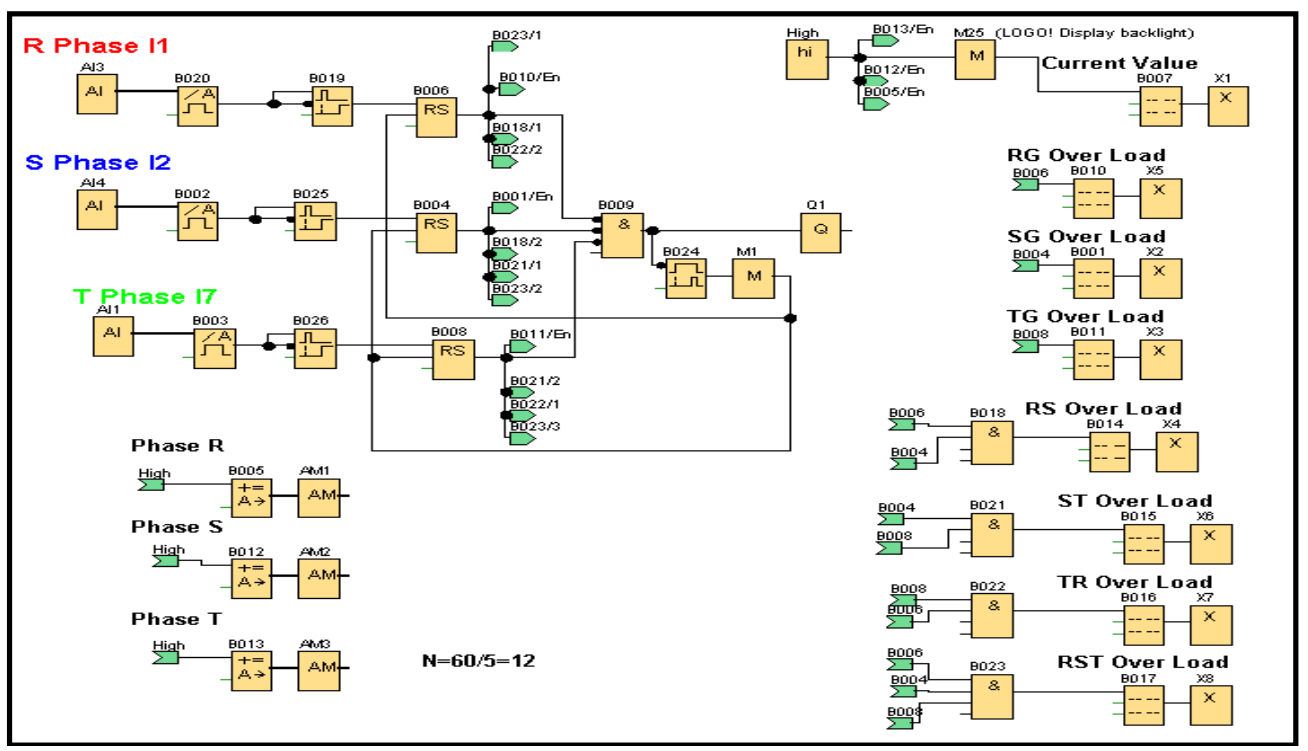

Fig. 3: FBD of the proposed protection control system

- Three of PLC's inputs are utilized (AI1, AI3, and AI4) are analog type, each one used for receiving the calibrated signal from $\mathrm{CT}$.

- Three Analog threshold trigger modules are used (B020, B002, and B003). The output of these modules is set (logic 1) depending on the thresholds value witch calibrated to the overload current.

- Three (RS) Latching relays (B006, B004, and B008) are used for latching the signal of the threshold trigger module when faults have occurred.

- AND gate (B009) with inverted inputs is used to activate the output of the (Q1) for all types of faults that occur at any phase.

- One of PLC's output modules is utilized (Q1), used for activating the trip coil of main CB.

- Three Analog Mathematics Instruction function blocks (B005, B0012, and B013) are utilized for finding (or calculating) the load current of each phase according to the following formula:

Load Current $=\frac{\text { Analog signal value }}{100} * R_{\text {Sh }} * \mathrm{~N}$

Where $\mathrm{N}$ is the turn ratio of the $\mathrm{CT}$.

- Three of Retentive on-delay Timer (B019, B025, and B026) are used to prevent tripping the $\mathrm{CB}$ during the starting the loads via delaying the overcurrent signals which sent from Analog threshold trigger modules to Latching relays with definite time (4second).

- On-delay Timer (B024) is used for reclosing the CB after definite time by resetting the Latching relays (i.e. On-delay timer (B024) that's output is not switched on until a configured delay time is expired, and it is used for reclosed the trip coil of the $\mathrm{CB}$ to the work after 10 cycles $(2 \mathrm{~ms})$ if the load current is returned to the value below than the threshold value or after the faults are released).

- Eight message texts are used for displaying the parameter of the system.

- First message displayed the values of the load current of each phase, which calculated using Analog mathematic instruction function block according to equation (1). 
- Three next message text show the name of the phase that overloaded with (Line to Ground) fault as following: "RG Over Load" message when phase R is overloaded, "SG Over Load" message when phase $\mathrm{S}$ is overloaded, "TG Over Load" message when phase T is overloaded.

- The second three message text show the name of the two phases that are overloaded with (Line to Ground) fault as following: "RG Over Load" message when phase R is overloaded, "SG Over Load" message when phase $\mathrm{S}$ is overloaded, "TG Over Load" message when phase $\mathrm{T}$ is overloaded.

- $\quad$ The last message is "RST Over Load" when three-phase are overloaded simultaneously.

\section{Simulation and Experimental Results}

The proposed FBD control program is tested using LOGO! Soft Comfort Simulation property; then program is downloaded from the PC into PLC's memory through special cables called (PC - LOGO! cable). Different faults are simulated to show the activation the output module (Q1) with addition to the displaying message text at each case: figure 4 shows the output signal (Q1) is high which indicate the CB is on state (on live) before occurring any faults (don't exceed the threshold values), see the message text which shows the amplitude of the load current for each one of three phases.

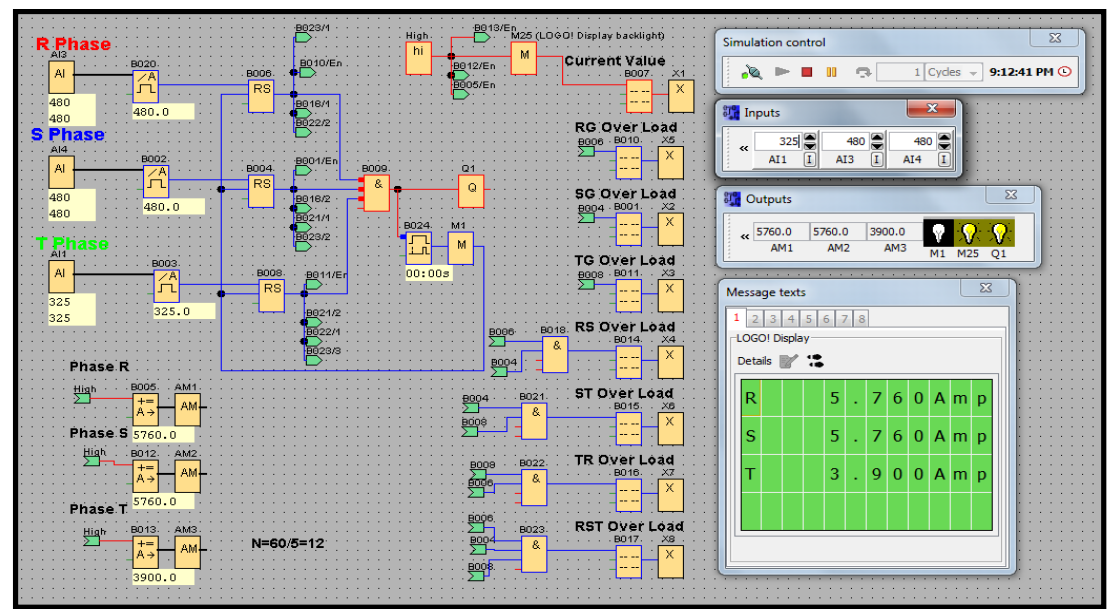

Fig. 4: Simulation circuit of the proposed FBD program

Figure 5 shows the simulation circuit of the system under phase $\mathrm{R}$ is overloaded by line to ground fault, see the "RG Over Load" message appears on PLC display unit. Figure 6 shows the simulation circuit of the system when two phases ( $T \& R$ ) are overloaded by line to line fault, see the "TR Over Load" message appear on PLC display unit. Figure 7 shows the simulation circuit of the system when three phases $(R, S \& T)$ are overloaded by line to line to line fault, see the "RST Over Load" message appear on PLC display unit. See the output (Q1) is inactive (i.e. $\mathrm{CB}$ is open) under all mentioned faults. 


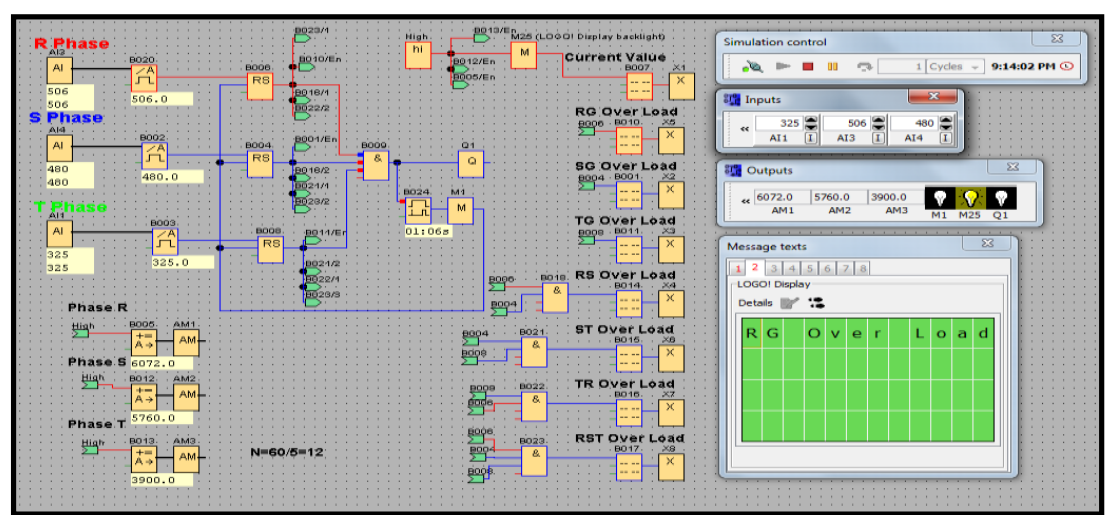

Fig. 5: Simulation circuit for L-G fault

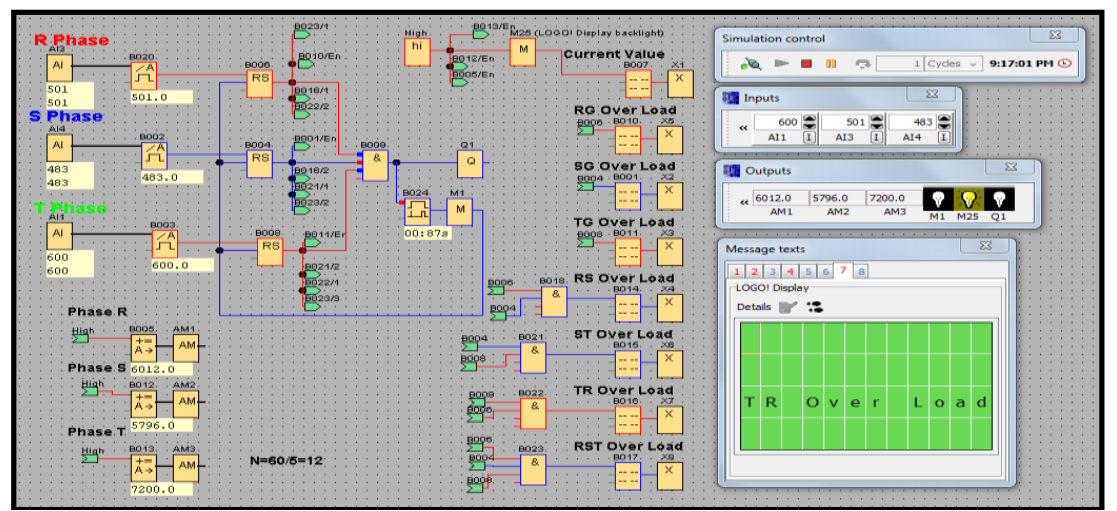

Fig. 6: Simulation circuit for L-L fault

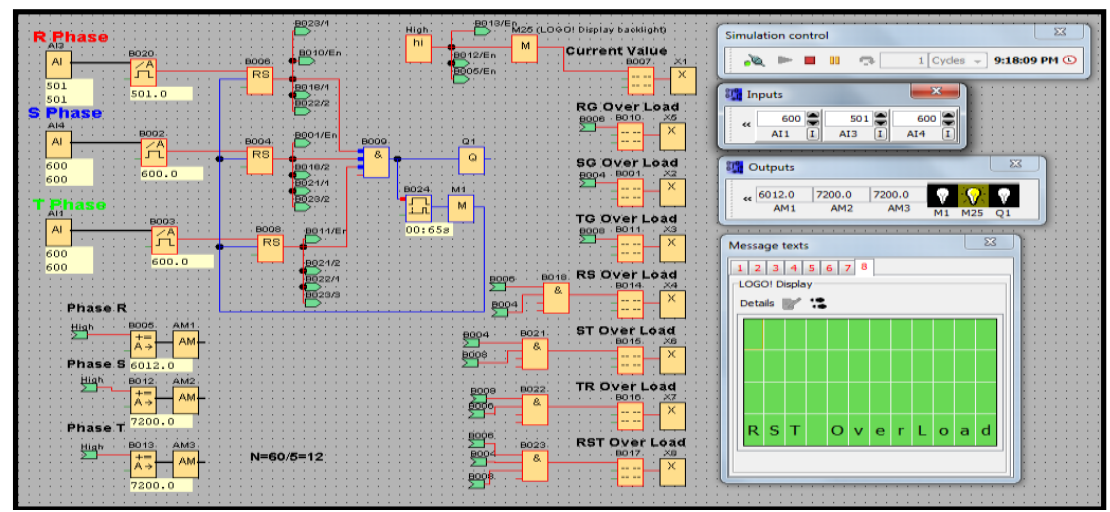

Fig. 7: Simulation circuit for L-L-L fault

Figure 8 shows the message texts (on-line) on the display unit of the PLC. Figure 8 (a) shows the amplitude of the load current for each one of three phases. Figure 8 (b) shows the message under phase $\mathrm{R}$ is overloaded by line to ground fault, see the "RG Over Load" message 
appear on PLC display unit. Figure 8 (c) show the message under when two phases $(\mathrm{T} \& \mathrm{R})$ are over loaded by line to line fault, see the "TR Over Load" message appear on PLC display unit. Figure 8 (d) shows the message when three phases (R, S \& T) are over loaded by line to line to line fault, see the "RST Over Load" message appear on PLC display unit.

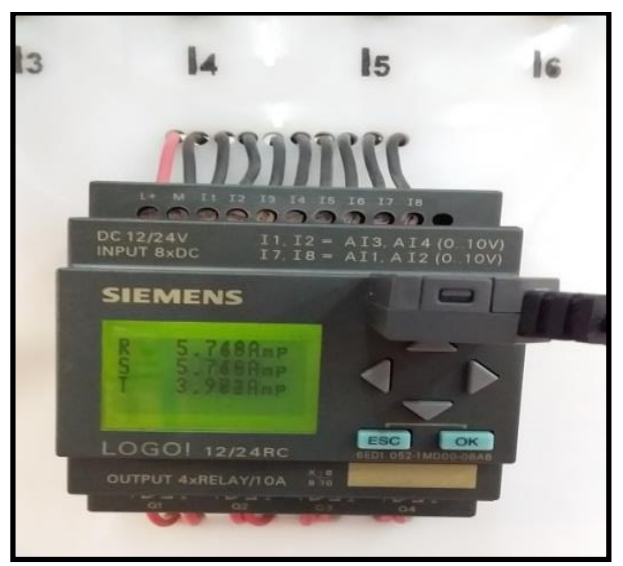

(a): Message text for values of three current

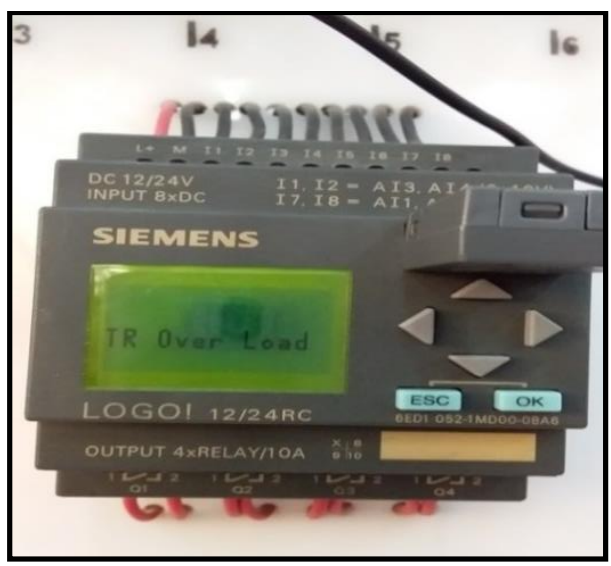

(c): Message text for L-L fault

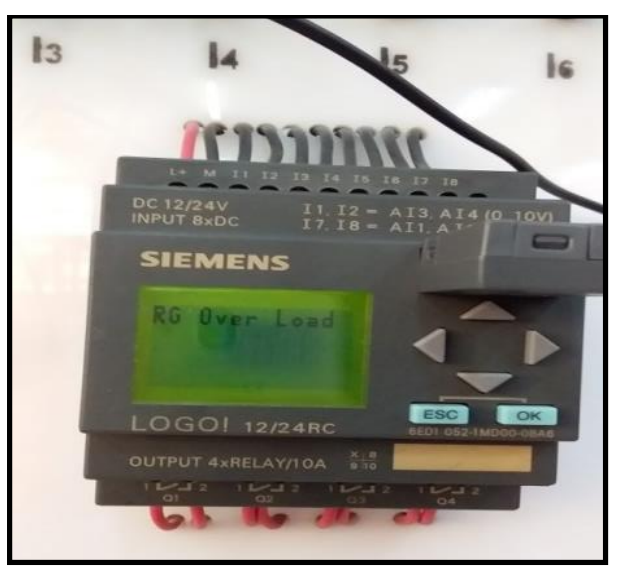

(b): Message text for L-G fault

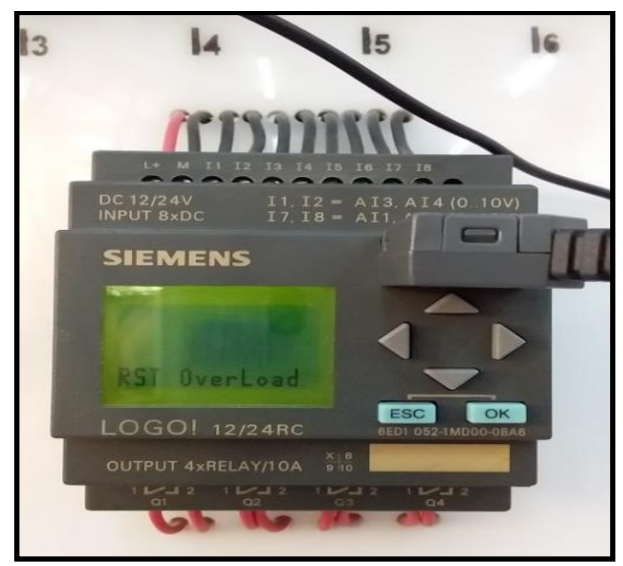

(d): Message text for L-L-L fault

Fig. 8: Message text on the PLC display unit

\section{Conclusions:}

- Experimental results present that the proposed protection system based on PLC has cost less, robust and high durability, offers higher precision in addition to safe and visual environment related to the classical.

- The proposed protection system operate to monitor the amplitude of the load current via calibration equation depending on the value of (shunt resistor, analog value, and the turn 
ratio of the $\mathrm{CT}$ ), then detect the overload current and give the output control signal to the CB.

- The data of the system: value of the load current and type of faults displayed on the PLC display unit in addition to displaying these data on the PC using" Online Test" feature in the in LOGO! Soft Comfort.

- The proposed overcurrent protection system has a property of cancelation the trip signal of the $\mathrm{CB}$ during a definite time of the inrush and stating load current, in addition to reclosing the $\mathrm{CB}$ after release the faults.

- It's easy to variation the time setting of the timers which are used in the proposed system to obtain the desired operating characteristics and increase the sensitivity of the protection system.

- The utilization of the employed OBA6 PLC resources (as Function Blocks, Digital Inputs, Digital Outputs, Flags, Program memory and other) for this work is very low, its possible in future work modifying this work and adding other sensors as voltage and implementation another protection system as distance and differential protection system for power system station.

\section{References:}

[1] R. Agrawal, and E. Koley, "Fuzzy Logic Based Protection Scheme for Symmetrical and Unsymmetrical Faults in Three Phase Series Compensated Transmission Line", International Conference on Micro Electronics and Telecommunication Engineering, Ghaziabad, 2016, PP. 471-475. doi: 10.1109/ICMETE.2016.88.

[2] Abhishek Pawar, Karishma Pawar, Prof. Piyush Desai, "Fault Detection of Induction Motor by Using PLC", HCTL Open International Journal of Technology Innovations and Research, 2017, Vol. 24, Issue 2, PP. 1-7.

[3] L. Krishnan, A. T. Pandian, R. Vijayakumar, "Over Current Protection of 1 KVA Transformer With PIC Micro Controller Using Numerical Relay", International Journal of Scientific \& Engineering Research, 2015, Vol. 6, Issue 2, PP. 948-954.

[4] Harsh Dhiman, "Design Analysis and Realization of Microcontroller Based Over Current Relay with IDMT Characteristics: A Proteus Simulation", International Journal of Science, Engineering and Technology, 2015, Vol. 3, Issue 5, PP. 1146-1150.

[5] S. Makwana and V. Makwana, "Simulation and Hardware Implementation of Over-current Relay Used for Transmission Lines", $3^{\text {rd }}$ International Conference on Trends in Electronics and Informatics, Tirunelveli, India, 2019, PP. 1189-1193. doi: 10.1109/ICOEI.2019.8862569.

[6] K. Shehata, A. Bahaa, K. Morad and A. Sharaf, "Design and Implementation of FPGA Based and Microcontroller Based Current Relay," Proceedings. The 16th International Conference on Microelectronics, Tunis, Tunisia, 2004, PP. 783-786. doi: 10.1109/ICM.2004.1434783

[7] G. P. H. Sandaruwan, P. S. Ranaweera and V. A. Oleshchuk, "PLC Security and Critical Infrastructure Protection", IEEE $8^{\text {th }}$ International Conference on Industrial and Information Systems, Peradeniya, 2013, pp. 81-85. doi: 10.1109/ICIInfS.2013.6731959.

[8] Ahmed M. T. Ibraheem, Omar Talal Mahmood Altaee, Noha Abed-Al-Bary Al-jawady, "PLC Controlled Multiple Stepper Motors Using Various Excitation Methods", IEEE International Conference on Engineering Technologies and their Applications, Islamic University - ALNajaf - Iraq, 2018, PP. 54-59. doi: 10.1109/IICETA.2018.8458097. 